\title{
(Un-)Sicherheit und Grenzen
}

\section{Alexandra Schwell}

\begin{abstract}
Der Beitrag argumentiert aus einer kulturanthropologischen Perspektive, dass Grenzen und Grenzziehungen für das Verständnis von Sicherheit und Unsicherheit von zentraler Bedeutung und zugleich eng mit der Vorstellung vom Nationalstaat als Sicherheitsgemeinschaft verknüpft sind. Grenzen sind nicht selbstevident und objektiv vorhanden, sondern sozial konstruiert und performativ. Das Verhältnis von Grenzen und (Un-)Sicherheit ist stets relational und muss im Kontext betrachtet werden, um die Komplexität der Vielzahl von Akteur"innen und Aktanten miteinzubeziehen, welche die Bedeutung von Grenzen in der gelebten Praxis prägen.
\end{abstract}

\section{Schlagwörter}

(Un-)Sicherheit, Grenzkontrolle, Souveränität, Nationalstaat, Migration

\section{Einleitung}

In diesem Beitrag soll der Zusammenhang von Grenzen und (Un-)Sicherheit aus kulturanthropologischer Perspektive diskutiert werden. Es soll davon ausgegangen werden, dass Grenzen und (Un-)Sicherheit untrennbar miteinander verbunden sind. Wer über Grenzen spricht, spricht zugleich implizit über Sicherheit und Unsicherheit. Dabei geht es nicht allein um den offensichtlichen Zusammenhang, der sich in den Themen Grenzsicherung und Grenzschutz äußert. Auch wenn die Bedeutung des Zusammenhangs von Grenze und (Un-)Sicherheit alltagspraktisch relevant, medial präsent und Gegenstand scharfer politischer Auseinandersetzungen ist, so äußert sich hier nur einer von mehreren Aspekten des Komplexes von (Un-)Sicherheit und Grenze. In diesem Sinne versteht dieser Beitrag Grenzsicherheit „as embodied through rare forms of knowledge that are produced, shaped, circulated, and consumed in and through practice“ (Baird 2017, S. 189).

Dieser Beitrag will die hier genannten verschiedenen Aspekte des Zusammenhangs von Grenze und (Un-)Sicherheit ideengeschichtlich herleiten und auf diese Weise wiederum die besondere sicherheitsrelevante Bedeutung, die Grenzen zukommt, beleuchten. Eine kulturwissenschaftliche Perspektive auf Grenzen betont dabei den Konstruktionscharakter beider Konzepte und weist zugleich auf ihre praktische Wirkmacht in Bezug auf Handlungsmöglichkeiten sowie die alltagspraktische Bedeutung von Selbst- und Fremdwahrnehmungen hin. Ein weiter Begriff von Grenze und (Un-)Sicherheit, der von der Lebenswirklichkeit von Akteur*innen ausgeht, steht dabei einer staatszentrierten und positivistischen Vorstellung von Grenzen und (Un-)Sicherheit gegenüber, wie er häufig in den Politikwissenschaften oder Teilen der Geografie gebraucht wird. Dagegen eröffnet der in diesem Text zum Tragen kommende weite Fokus eine Perspektive auf die Vielfalt der Räume, Praktiken, Diskurse und entsprechende Kontingenzen und trägt damit zu einem umfassenderen Verständnis des Zusammenhangs von (Un-)Sicherheit und Grenzziehungsprozessen bei.

In einem ersten Schritt wird in der Einleitung der Zusammenhang von Grenzen und (Un-)Sicherheit im Hinblick auf kollektive Identitätsprozesse herausgestellt. Dabei werden auch die Begriffe Grenze und Sicherheit kontextualisiert und definiert. In einem zweiten Schritt wird auf 
dieser Grundlage gezeigt, wie die Sicherung nationalstaatlicher Grenzen eine Voraussetzung für die Vorstellung einer nationalstaatlichen Gemeinschaft darstellt. Es werden exemplarisch Institutionen und Handelnde des Komplexes Grenzen-(Un-)Sicherheit vorgestellt. Dabei wird deutlich, dass das Verhältnis von Grenzen und (Un-)Sicherheit stets relational ist und kontextual betrachtet werden muss, um die Komplexität der Vielzahl von Akteur"innen und Aktanten miteinzubeziehen. Schließlich handeln nicht nur Menschen an und mit Grenzen, sondern auch Technologien, Politiken, Materialitäten und Infrastrukturen spielen eine wichtige Rolle (vgl. Aradau 2010; Carey/Pedersen 2017).

\subsection{Grenzen und kollektive Identitäten}

Grenzen wird die inhärente Funktion zugeschrieben, Sicherheit für Individuen und soziale Gruppen zu stiften. Sicherheit bezieht sich dabei sowohl auf das persönliche Wohlergehen und Unversehrtheit (safety) wie auch auf die institutionell oder politisch geregelte Sicherheit (security; zur Unterscheidung vgl. Eisch-Angus 2011). Sicherheit und Eindeutigkeit beginnen bereits auf der Mikroebene: Identitätsprozesse basieren auf Grenzziehungen. Erst durch Grenzziehungen kann Differenz entstehen, können Unterscheidungen und Kategorisierungen getroffen werden. Und erst durch Grenzziehungen können Wir uns von den Anderen unterscheiden und etwas Eigenes definieren. Grenzen sind deshalb untrennbar mit kollektiven Identitäten verbunden. Bereits Max Weber definierte die Anziehung nach innen und die Abstoßung nach außen, also die Grenzziehung zwischen dem Eigenen und dem Fremden, als Merkmale ethnischer Vergemeinschaftung (vgl. Weber 1921/2005, Kap. IV). Fredrik Barth (1969) zeigt in seinem Werk Ethnic Groups and Boundaries. The Social Organization of Cultural Difference auf, dass Identität nicht so sehr, wie häufig angenommen, darauf basiert, dass Kollektive glauben, etwas gemeinsam zu haben. Vielmehr argumentiert er, dass in erster Linie die Art und Weise, wie die Grenze zu den Anderen, den Nichteigenen, gezogen wird, für die Herausbildung einer gemeinsamen Identität verantwortlich sei. Grenzen dienen in diesem Sinn zur Identitätsversicherung und Selbstversicherung. Herauszufinden, wer Wir sind, wird erleichtert, wenn ich weiß, was Uns von den Anderen unterscheidet.

Das Deutsche kennt im Unterschied zu anderen Sprachen lediglich ein Wort für die Grenze. Im Englischen finden mehrere Begriffe Verwendung, die auf die jeweils unterschiedlichen Funktionen von Grenzen verweisen. In den Kulturwissenschaften und der Kulturanthropologie wird die nationalstaatliche Grenze häufig als border bezeichnet, als die Grenze, die Gesetzgebungen und souveräne Nationalstaaten voneinander trennt. Die boundary hingegen wird eher mit der symbolischen, kulturellen und sozialen Grenze in Zusammenhang gebracht. Anthony P. Cohen (1998, S. 26) definiert boundaries als

„subjects of claim based on a perception by at least one of the parties of certain features which distinguish it from others. Whether it refers to a collective condition, such as ethnic group identity, or to something as ephemeral as ,personal space‘, boundary suggests contestability, and is predicated on consciousness of a diacritical property“.

Nationalstaatliche Grenzen stellen zwar immer auch soziale Grenzen dar, kulturelle oder sprachliche Grenzen müssen jedoch nicht mit nationalstaatlichen Grenzen zusammenfallen. Das nationalstaatliche Projekt des sogenannten Westfälischen Staatensystems strebt danach, politische Grenzen mit kulturellen, sprachlichen und anderen, wie religiösen, Grenzen in Ein- 
klang zu bringen. Anthony P. Cohen (1998, S. 29) stellt entsprechend fest: „The border is a social fact. Whether or not it signifies difference is a matter of social construction and is more properly thought if as one of boundary“. Identität, so Cohen weiter, sei durch Unsicherheit motiviert und berge die Sehnsucht nach Sicherheit und Gewissheit in sich.

Wie wichtig Grenzziehungen für die Sicherung kollektiver Identitäten sind, zeigen die Forschungen der Ethnologin Mary Douglas. In ihrer Untersuchung Purity and Danger (Douglas 1966/1984) argumentiert sie, dass Kollektive klare Grenzziehungen und Unterscheidungen einfordern. Verschmutzung werde als Grenzverletzung und damit zugleich als Bedrohung einer symbolischen Ordnung, als Gefahr für das eindeutige Eigene interpretiert. „Dirt“, so ihr berühmtes Diktum, sei „matter out of place“ (ebd., S. 36). Diese eingängige Definition impliziere laut Douglas (ebd., S. 36f.) zwei Bedingungen:

„a set of ordered relations and a contravention of that order. Dirt then, is never a unique, isolated event. Where there is dirt there is system. Dirt is the by-product of a systematic ordering and classification of matter, in so far as ordering involves rejecting inappropriate elements. This idea of dirt takes us straight into the field of symbolism and promises a link-up with more obviously symbolic systems of purity.“

Douglas sieht ihre Erkenntnisse über den religiösen Kontext hinaus als relevant an und begreift Grenzen jeglicher Art als höchst bedeutsam für kulturelle und soziale Prozesse in einem weiteren Sinn. Ihre Vorstellung von Grenzverletzungen und „matter out of place“ haben insbesondere im Bereich der Migrationsforschung zahlreiche Studien inspiriert (Ahmed 2000; Chavez 2007; Brubaker 2010). Illegalisierte Migration wird auf diese Weise nicht allein als Grenzverletzung, sondern als regelrechte Verschmutzung einer als rein deklarierten Gemeinschaft gedeutet. Auch Kosmopolitismus, Hybridität und Fluidität verweisen auf Uneindeutigkeit, potenziell zweifelhafte Loyalität und Subversion und deshalb auf Unsicherheit (vgl. Bhabha 1994). Klare Grenzen dagegen schaffen Eindeutigkeit und produzieren Sicherheit, indem sie Eigenes, Bekanntes und deshalb auch Vorhersehbares vom Fremden, Unbekannten und Unberechenbaren unterscheiden.

Gemeinschaften schützen ihre boundaries auf unterschiedliche Weise, durch religiöse und kulturelle Praktiken, sprachliche Grenzziehungen, historisch tradierte Narrative, Tabus oder gemeinsame Symbole. Diese Vorstellung von der Grenze, die vorgeblich reine Entitäten voneinander trennt und deren Vermischung, Überlappung und Grenzverletzung eine Verunreinigung bedeuten, verdeutlicht, warum sich dieses Denkmodell als so außerordentlich erfolgreich für die Konzeption des Nationalstaates erwiesen hat. Laut Benedict Anderson (1998) ist eine Nation kein objektives Gegebenes, sondern eine „vorgestellte Gemeinschaft“ - wie im Übrigen jede Gemeinschaft, in der sich nicht alle Mitglieder persönlich kennen. Die Vorstellung einer reinen nationalen Gemeinschaft muss entsprechend auf der Vorstellung eindeutiger Grenzen dieser Gemeinschaft basieren, um politisch mobilisierungsfähig zu sein. Aber was bedeutet Sicherheit?

\subsection{Sicherheit}

Eine sehr allgemein gehaltene Definition setzt Sicherheit mit der Abwesenheit von Bedrohung gleich (Booth 1991, S. 319). Allerdings ist Bedrohung kein objektiver Begriff; entsprechend kann Sicherheit an unterschiedlichen Orten zu unterschiedlichen Zeiten für verschiedene Indi- 
viduen und soziale Gruppen jeweils andere Bedeutungen annehmen. Sicherheit ist demnach relational, da sie nicht objektiv existiert, sondern stets auf etwas Anderes verweist. Sicherheit bezieht sich immer auf ein Referenzobjekt (Was oder wer muss geschützt werden?) und ein Referenzsubjekt (Wer oder was ist ein Sicherheitsproblem?). Die vorherrschenden Sicherheitsdiskurse und Sicherheitsprobleme innerhalb einer Gesellschaft müssen also nicht identisch sein; zugleich ist eine kultur- und sozialwissenschaftliche Analyse derselben aufschlussreich, um mehr über die Lebenswirklichkeiten und ihre Kontingenzen zu erfahren (Furedi 2006; Gusterson/Besteman 2010; Schwell/Eisch-Angus 2018).

Trotz aller Unschärfe kann argumentiert werden, dass es durchaus ein individuelles und kollektives Grundbedürfnis nach Sicherheit gibt. Sicherheit ist etwas, nach dem Menschen gesellschaftsübergreifend streben und das sie wertschätzen, unabhängig von ihrer Gesellschaftsform, Kultur oder Schichtzugehörigkeit. Im Umkehrschluss kann Unsicherheit sich als destruktiv erweisen, für das menschliche Dasein wie auch für die persönliche und soziale Entwicklung. Ohne diese grundlegende Sicherheit sind psychische und physische Entwicklungs- und Entfaltungsmöglichkeiten eingeschränkt. Anthony Giddens (1990, S. 92) spricht von „ontological security“ als emotionalem Phänomen, als „the confidence that most human beings have in the continuity of their self-identity and in the constancy of their surrounding social and material environments of action“. Dazu gehören die Vorhersehbarkeit und Planbarkeit, die Gewissheit, dass die Welt morgen noch so sein wird wie heute.

Unsicherheit im Sinne von Prekarität und „Risikogesellschaft“ (Beck 1986) umfasst laut dem Soziologen Zygmunt Bauman (2000, S. 161; Herv. i. O.) drei Dimensionen: „insecurity (of position, entitlements and livelihood), of uncertainty (as to their continuation and future stability) and of unsafety (of one's body, one's self and their extensions: possessions, neighbourhood, community)." Für Bauman sind diese Formen der Unsicherheit Kennzeichen einer zunehmend unübersichtlich werdenden und entgrenzten Welt. Status, Auskommen, Beruf, Lebensplanung sind Gegenstand von Unsicherheiten und entsprechend von Bemühungen der Grenzsicherung. Die Vorstellung festgelegter, unverhandelbarer und undurchdringbarer borders und boundaries erweist sich insbesondere in Transformations- und Umbruchzeiten, die als unsicher empfunden werden, als wirkmächtig. Eine Verletzung dieser imaginierten Grenzen hat entsprechend Folgen für das subjektive Sicherheitsgefühl, und dies in sozialer wie politischer Hinsicht.

Da Sicherheit relational ist, sind stets unterschiedliche Akteur*innen beteiligt. So konstituiert der Rekurs auf Sicherheit und Sicherheitsmaßnahmen ein Objekt in zweifacher Hinsicht: Erstens konstituiert der Diskurs etwas als schützenswert und zweitens als bedroht und potenziell verwundbar. Mit diesem Objekt korrespondiert eine als solche deklarierte Bedrohung. Verbunden ist die Vorstellung eines Zukunftsszenarios (vgl. Appadurai 2013), dessen Immanenz den politischen Akteur"innen helfen soll, die Dringlichkeit der Bedrohung zu verdeutlichen und die Einleitung entsprechender Gegenmaßnahmen durchzusetzen. Dieser Prozess wird als securitization, Versicherheitlichung, definiert und betont die Prozesshaftigkeit und den Konstruktionscharakter von Sicherheitsbedrohungen und -maßnahmen sowie ihre inhärenten Machtasymmetrien (vgl. Buzan et al. 1998; Balzacq 2011).

Sicherheit ist also nicht allein eine soziale Konstruktion, sondern auch ein wirksames Machtinstrument. Michel Foucault unterscheidet Sicherheitsmaßnahmen klar von Disziplinarmaßnahmen, wobei erstere sich dadurch auszeichnen, dass sie nicht auf totale Kontrolle und Überwachung ausgerichtet sind, sondern Unsicherheit explizit eingeplant wird, indem die Grenzen des Akzeptablen definiert werden (vgl. Foucault 2006, S. 20). Sein Begriff des Sicherheitsdispo- 
sitivs wird auch in Forschungen zu Grenzen und Grenzsicherheit, insbesondere in Bezug auf Migration und Kriminalität, aufgegriffen, weil damit die Filterfunktion der staatlichen Grenze sowie das Verhältnis von Mobilität und Grenzfunktion gut beschrieben werden können (vgl. Hess/Kasparek 2010; Buckel 2013).

\section{Die Sicherung der nationalstaatlichen Grenzen}

Im Nationalstaat kommen diese beiden Konzepte, Grenzen und Sicherheit, auf spezielle Weise zusammen. Dies hat mit einer besonderen Qualität des Nationalstaats zu tun, die über seine nüchterne völkerrechtliche Definition hinausgeht. Mit der Entwicklung des Nationalstaats seit dem 18. Jahrhundert ging auch eine emotionale Aufladung einher, eine Nationalisierung von Identitäten, die zahlreiche Bereiche des öffentlichen und privaten Lebens umfasste (vgl. Weber 1976; Löfgren 1989; Billig 1995; Götz 2011) und eine nationale Zugehörigkeit erschuf. Der Nationalstaat wird so zum Ort der emotionalen Beheimatung, zur Heimat.

\subsection{Der Nationalstaat als emotionale Beheimatung}

Daheim, Zuhause und Heimat sind symbolhafte Begriffe für Sicherheit, Wärme und Zuflucht vor den Gefahren dieser Welt. Das Projekt des Nationalismus und Nation-Building-Prozesse waren auch deshalb so außerordentlich erfolgreich, weil der Nationalstaat sich als Heim für eine vorgestellte Gemeinschaft, sozusagen die Familienmitglieder, darzustellen wusste und weiß (vgl. Anderson 1998). Der Nationalstaat ist die primäre Sicherheitsgemeinschaft auf gesellschaftlicher Ebene, und dies in vielerlei Hinsicht: Er verfügt über das Gewaltmonopol, er schützt die Grenzen gegen äußere Eindringlinge, er sorgt für die Aufrechterhaltung der öffentlichen Ordnung, er verteidigt das Innere in Kriegszeiten, er baut Straßen, Schulen, Krankenhäuser - und all diese Faktoren tragen zu dem Gefühl bei, Teil einer Sicherheitsgemeinschaft zu sein. Interessanterweise fungiert der Staat selbst dann als Heim, wenn er seine Aufgaben nicht entsprechend der Vorgaben erfüllt. Wie Michael Herzfeld (1997) am Beispiel Griechenlands zeigt, kann dieses nationalstaatliche Zuhause auch durchaus ein Ort sein, den man zutiefst verachtet oder dessen man sich schämt.

Es liegt in der Verantwortung des Staates, für seine Bevölkerung Sicherheit zu gewährleisten. Analog zum Zusammenhang von Grenzziehungen und kollektiven Identitäten sind nationalstaatliche Grenzen dabei konstitutiv und grundlegend für die Imagination des Nationalstaates als Heim und der Definition und der Abgrenzung vom bedrohlichen Fremden. Diese Domopolitics (Walters 2004; Darling 2011, 2014), hängen eng mit der Bedeutung von Grenzen zusammen. Mit „Domopolitics“ bezeichnet Walters (2004, S. 241) Regierungspraktiken, die den Nationalstaat als Heim erscheinen lassen: „If modern political economy echoes the project of government in the image of the household, domopolitics refers to the government of the state (but, crucially, other political spaces as well) as a home [Herv. i. O.]“. Dabei, so Walters (ebd., S. 241), richten „Domopolitics“ das Verhältnis von Citizenship, Staat und Territorium neu aus:

„At its heart is a fateful conjunction of home, land and security. It rationalizes a series of security measures in the name of a particular conception of home. [...] Hence domopolitics embodies a tactic which juxtaposes the ,warm words' (Connolly 1995, S. 142) of 
community, trust, and citizenship, with the danger words of a chaotic outside - illegals, traffickers, terrorists. “

Dabei wird deutlich, dass Grenzsicherung stets eine externe Bedrohung für ein schützenswertes Inneres impliziert. Hier begegnen wir Max Webers grundlegender Funktion von Grenzziehungen für kollektive Identitätsprozesse und Selbstversicherungen wieder, die im Rahmen des Nationalstaats politisiert und national aufgeladen wird. Entsprechend weist Nicholas De Genova (2010, S. 49) auf die Bedeutung dieser Relationalität für unbewusste Vorstellungen vom Eigenen und Fremden und deren Einfluss auf politisches Denken hin:

"Thus the pervasive assumption of a polity of citizen-comrades who inhabit a ,domestic space, always starkly demarcated from an amorphous ,foreign' exterior, is symptomatic of $[\ldots]$ 'the geographical unconscious' and stands as one of the enduring effects of ,the territorial trap" of contemporary political thought."

Laut Weber (1921/2005, S. 1043) bedeutet staatliche Souveränität, dass ein Staat über das Gewaltmonopol verfügt, indem er die soziale Ordnung innerhalb eines gegebenen Territoriums über eine definierte Bevölkerung aufrechterhält und indem er zugleich die Grenzen nach außen schützt. Eine der wichtigsten symbolischen Funktionen der Nationalstaatsgrenze basiert auf der Tatsache, dass Grenzen der Bevölkerung des Territoriums, das sie umgeben, Sicherheit versprechen. Grenzkontrollen und die Idee des Nationalstaats hängen unauflösbar miteinander zusammen. Grenzkontrollen verkörpern sozusagen den Gründungsmythos des Nationalstaates. Sie sind einer der Hauptgründe, warum der Grenzübertritt für den Nationalstaat ein Machtinstrument ist und der Nationalstaat zugleich an seinen Grenzen am sichtbarsten und erfahrbarsten ist.

Ein Hauptzweck von Grenzen besteht darin, Mobilitäten zu kategorisieren und zu klassifizieren. William Walters (2006a, S. 197) hält diese „sorting function“ für eine immer zentraler werdende Funktion der Grenze. Ob Tourist*innen, Migrant*innen, Asylsuchende, Diplomat*innen, kurz, ob Grenzgeher*innen als erwünscht oder unerwünscht klassifiziert werden, liegt in der Hand des Nationalstaates, den sie betreten. Hinzu kommen internationale Verträge und Verpflichtungen wie die Genfer Flüchtlingskonvention oder Abkommen, die die Behandlung diplomatischen Personals regeln. Diese vielfältigen und gleichzeitigen Grenzziehungsprozesse, die auch als bordering (vgl. Houtum/Naerssen 2002) bezeichnet werden, markieren Körper als zugehörig oder illegal. Die Grenze existiert entsprechend für unterschiedliche Mobilitäten und Akteur*innen auf unterschiedliche Weise: Sie funktioniert weniger als undurchdringbare Festung, wie es das Schlagwort von der Festung Europa will, sondern als Filter, als durchlässige „Membran“ (Walters 2004) oder als „Firewall“ (Walters 2006b), die unerwünschte Eindringlinge herausfiltert (vgl. auch Vollmer/Düvell in diesem Band).

Dabei sind Grenzsicherheit und Grenzkontrollen historisch recht neue Erfindungen. Während ökonomische Zollgrenzen historisch weit zurückreichen, wurden Reisepässe erst mit dem Ersten Weltkrieg flächendeckend in Europa eingeführt (vgl. Torpey 2000). In den USA wurden bis in die 1820er Jahre keine Einreiseregister geführt, bis in die 1880er Jahre unterlag Immigration keiner bundesstaatlichen Regelung (Walters 2004, S. 250). Die seither immer umfassendere Ausweitung und Verfeinerung der Grenzsicherheitsmechanismen hat nicht allein etwas mit zunehmenden technischen Möglichkeiten zu tun, sondern zeigt, dass Grenzsicherheit in ein weiter reichendes System von Kontrolle und Biomacht eingebettet ist, in ein Sicherheitsdispo- 
sitiv, dessen Ziel die, im wörtlichen Sinn, Unterwerfung des Subjekts, die Verwaltung, das Regieren, das Management von Körpern und Bevölkerungen sind.

\subsection{Institutionen der Grenzsicherung}

Grenzen existieren nicht einfach, sondern sind das Ergebnis von Grenzziehungsprozessen. In diesem Abschnitt soll nun ein Blick auf diejenigen Institutionen und institutionellen Akteur*innen der Grenzsicherung geworfen werden, die mit diesen bordering processes verbunden sind, und auf die Prozesse, die von ihnen ausgehen. Es wurde bereits auf die Bedeutung von Narrativen, symbolischen und kulturellen Grenzziehungen sowie politischen und rechtlichen Grenzen hingewiesen. Grenzen sind stets performativ und damit in der Lage, Realitäten verändern zu können (vgl. Andreas 2001). Chris Rumford (2008) geht davon aus, dass Grenzen nichts Statisches und Selbstevidentes sind, sondern das Ergebnis aktiver „Borderwork“, an der nicht nur staatliche Stellen, sondern auch zivile oder ökonomische Akteur*innen beteiligt sind. Grenzen sind „enacted and performed not only as ,discursive or emotional landscapes of social power on the one hand, but also as ,technical landscapes of control and surveillance" on the other" (Johnson et al. 2011, S. 62).

Die Sicherung nationalstaatlicher Grenzen umfasst entsprechend vielfältige Prozesse, von der rechtlichen und gesetzlichen Lage, beispielsweise des Staatsangehörigkeitsrechts oder der Einwanderungs- und Asylgesetzgebung, über die politische nationale und transnationale Sicherheitsarchitektur sowie staatliche und private Organisationen bis hin zu polizeilichen Kompetenzen, Strategien und internationalen Kooperationen (vgl. Sheptycki 2002; Schwell 2008), den technischen Maßnahmen der Datenspeicherung und -erfassung (Broeders 2007), biometrischen und Überwachungstechnologien an smart borders sowie Infrastrukturen (vgl. Pötzsch in diesem Band). Technische, administrative und rechtliche Ressourcen und Mittel kontextualisieren nicht nur, sondern spielen eine Mittlerrolle für Praktiken der Grenzziehung, indem sie die Praktiken selbst sowie die dahinterliegenden Ideen und Imaginationen auf vielfältige Weise in andere Lebens- und Politikbereiche übersetzen.

Dabei handelt es sich nicht um totalisierende Regime: Grenzkontrollen, Grenzsicherungsmaßnahmen und -politiken bringen eine Vielzahl von Prozessen hervor, die von unintendierten Effekten über Subvertierungen bis hin zu Widerständigkeiten reichen können. Schmuggel, grenzüberschreitende Kriminalität und Schlepperei sind nur die medial augenfälligsten Erscheinungen, die ihre Existenz der Grenze verdanken, wie Małgorzata Irek (1998) in ihrer Ethnografie des „Schmugglerzugs“ zwischen Deutschland und Polen in den 1990er Jahren zeigt. Karolina Follis (2012) begibt sich an die polnisch-ukrainische Grenze und zeigt in ihrer Studie Building Fortress Europe die Gleichzeitigkeit von vielartigen Grenzprozessen auf, die eine sich verändernde Grenze als Ausschließungsmechanismus, aber auch als Verbindungselement etablieren. Unerwartete Effekte, staatliche Mechanismen, transnationale Vorgaben und Vorstellungen informieren die Grenzregion und formen das Leben und den Alltag der Akteur*innen auf beiden Seiten der Grenze und in den Institutionen im Kontext der EU-Erweiterung und der sie begleitenden Erweiterungs- und Sicherheitspolitiken. Zugleich durchbrechen diese Prozesse die idealtypischen Vorstellungen von der Souveränität des Nationalstaats an der Grenze.

In all diesen Fällen bedingen sich Mobilitäten und Grenzziehungen gegenseitig und stehen in einer Wechselbeziehung. Hilary Cunningham und Josiah Heyman (2004, S. 293) unterscheiden 
zwischen „enclosure“ als „social processes that delimit and restrict the movement of specific goods, people, and ideas“ und „mobility, the social processes that enable and induce such movements“. Mit diesem Kontinuum von enclosure und mobility weisen sie darauf hin, dass die tradierte und vermeintlich selbstverständliche Gegenüberstellung von sesshafter Normalität und Mobilität als soziale Wandlungsprozesse sozial konstruiert und empirisch wie historisch kaum haltbar ist:

„Both enclosure and mobility are defined against the other, hence reflecting our sense of borders as ongoing social processes governed through political, economic, and cultural struggles. Enclosure and mobility offer valuable tools for exploring the interplay of power, resources, and ideology in the contemporary world, especially (but not uniquely) at state borders" (ebd.).

Wie Heidrun Friese (2017) am Beispiel der sogenannten Flüchtlingskrise zeigt, wird Mobilität häufig als regelrecht pathologisch, als „Ungleichgewicht, als Unterbrechung gesellschaftlichen Beharrens“ (ebd., S. 35) konzeptualisiert. Sie schreibt: „Mobilität wird zur Ausnahme, ja mehr, zum feindlichen Einfall von Parasiten, die den gesunden, integren Volkskörper schwächen und die zu verjagen, abzuschieben, wegzuschaffen oder an der Grenze des Volkskörpers zu töten, populistische Stimme lautstark fordert“ (ebd.). Diese symbolische Aufladung der Grenze als Schutz der reinen vorgestellten Gemeinschaft vor mobiler Verschmutzung verdeutlicht, dass Grenzen mit weit mehr als nur mit Fragen der staatlichen Territorialität verbunden sind und deshalb stets in einem Spannungsverhältnis zur Frage stehen, wie Staatlichkeit erschaffen wird, sich konstituiert, herausgefordert und imaginiert wird (vgl. dazu Herrmann/Vasilache in diesem Band). Walters zieht eine direkte Linie zwischen nationalstaatlichen Beheimatungsstrategien, Grenzsicherheit und Mobilität und zeigt auf diese Weise die appellative Funktion der „Domopolitics: ,our' homes are at risk“ (Walters 2004, S. 247).

\subsection{Die Ubiquität der Grenzkontrolle und der Ausnahmezustand}

Grenzen befinden sich jedoch nicht allein an den Rändern des Nationalstaates. Grenzen zeigen sich nicht allein in Linien, Mauern, Zäunen, Barrieren und Schlagbäumen, sondern treten auch punktuell auf, beispielsweise als „ports of entry“ inmitten eines Staatsgebiets, wie im Fall internationaler Flughäfen (vgl. Salter 2008a; Herlyn/Zurawski 2015). Hinzu kommen sogenannte „Remote Controls“ (Guiraudon 2003), Prozeduren und Technologien, die der Grenzkontrolle vorgelagert sind oder fernab davon stattfinden. Visaregime sind das augenfälligste Beispiel (vgl. Bigo/Guild 2005). Unter remote controls können auch Verbindungsbeamt*innen oder im Ausland stationierte Polizist*innen gefasst werden, die dem Grenzübertritt vorgelagerte Kontrollen vornehmen.

Zudem delegiert der Nationalstaat die Sicherheit seiner Grenzen zunehmend an Dritte, die hoheitliche Aufgaben ausüben, ohne dafür entsprechend ausgebildet oder legitimiert zu sein. Angefangen von privaten Sicherheitsdiensten an Flughäfen, die schlecht bezahlt und unzureichend ausgebildet sind, bis hin zu Passkontrollen durch Fluggesellschaften, die belangt werden, sollten sie jemanden mit unzureichenden Papieren reisen lassen, wie im Fall von „carrier liability sanctions“ (Guiraudon 2006). Ganz abgesehen von der fehlenden Legitimation, die staatliche Akteur*innen auszeichnet, sind privatwirtschaftliche Unternehmen eher geneigt, ökonomischen Imperativen zu folgen, als sich an demokratischen Richtlinien zu orientieren, 
die weniger gewinnbringend erscheinen. Die Grenzschutzfunktion, die eigentlich dem souveränen Staat vorbehalten sein sollte, wird an private Akteur*innen ausgelagert.

Gregory Feldmans (2012) Studie The Migration Apparatus widmet sich diesem Politikfeld des europäischen Migrationsmanagements und zeigt in einer facettenreichen „non-local ethnography“, wie verschiedenste Kontexte, Akteur*innen, Bürokratien, Diskurse, Technologien und Logiken miteinander verwoben sind. Auch Ruben Andersson (2014) beschreibt in seiner Studie Illegality, Inc. auf eindrückliche Weise, wie verschiedene Akteur*innen an der Südgrenze Europas konzertiert die illegalisierte Migration bekämpfen und paradoxerweise auf diese Weise das Phänomen, dessen sie eigentlich Herr werden sollen, zugleich produzieren. In seiner Ethnografie der Alltagspraktiken von Akteur*innen des europäischen Grenzregimes widmet sich Andersson dieser von ihm sogenannten „illegality industry“ (ebd.), die illegale Migrant“innen als Kategorie und Phänomen überhaupt erst produziere. Er argumentiert, diese Illegalitätsindustrie bringe unterschiedlichste staatliche und nichtstaatliche Akteur*innen über der Kontrolle von Mobilitäten zusammen. Auf diese Weise produziere die Illegalitätsindustrie genau das, was sie eigentlich doch abschaffen sollte, nämlich illegale Migration. Infolgedessen wird mehr Geld in die Bekämpfung illegaler Migration investiert, was zugleich das Schreckgespenst der Sicherheitsbedrohung an der Grenze aufrechterhält und damit auch den Bedarf nach Verteidigung perpetuiert.

Wie diese Beispiele zeigen, hat sich auch die Grenzfunktion, die Ausübung der Grenze und der Grenzkontrolle, von den territorialen Grenzen des Staates wegbewegt und findet verstreut an durchaus heterogenen Orten statt (vgl. Walters 2006a). Die Grenzfunktion kann an jedem beliebigen Ort ausgeübt werden, mit jeder Dokumentenüberprüfung, jeder Unterscheidung zwischen Bürger*innen und Nichtbürger*innen, jeder Klassifizierung von Zugehörigkeit und Ausschluss. Étienne Balibar (2009) formuliert dementsprechend die These, die Grenze sei überall. Damit meint er, dass die Grenzpraktiken des souveränen Staates nicht mehr allein entlang politischer Staatsgrenzen existieren, sondern dass die Grenzfunktion losgelöst von dieser border ausgeübt werden kann. Beispiele sind Krankenhäuser und Wohlfahrtsorganisationen, die Personenüberprüfungen vornehmen, um entsprechende Berechtigungen zu prüfen. Grenzen sind deterritorialisiert und delokalisiert und auf diese Weise zugleich dem Körper der Nichtzugehörigen eingeschrieben.

Grenzsicherung wirft damit auch grundlegende Fragen nach der Verhandlung von Citizenship und Zugehörigkeit auf. Der Sozialanthropologe Shahram Khosravi (2010, S. 99) schreibt: „undesirable people are not expelled by the border, they are forced to be border [Herv. i. O.]." Er selbst, aus dem Iran geflüchtet und mittlerweile schwedischer Staatsbürger, beschreibt in seiner Autoethnografie, wie er bei der Einreise nach Großbritannien vom britischen Grenzschutz aufgrund von Racial Profiling festgehalten und befragt wurde. Seine schwedische Staatsbürgerschaft, so zeigt sich, schützt ihn nur bedingt vor Zuschreibungen als Sicherheitsbedrohung und Grenzverletzer durch den Nationalstaat, den er zu besuchen beabsichtigt (vgl. ebd., S. 97-99).

Ähnlich wie Khosravi argumentiert auch Mark Salter (2008b) mit Bezug auf Giorgio Agamben (1998), dass die Grenze den Ausnahmezustand verkörpere. Der Nationalstaat sei nicht nur an seiner Grenze am mächtigsten, sondern die nationalstaatliche Souveränität äußere sich genau in dem Spielraum der individuellen Person des oder der Grenzschützer*in, über das Schicksal der Grenzgänger*innen zu befinden. Diese Souveränität und Autorität des Nationalstaates wird in der Person des oder der Grenzschützer*in verkörpert. Salter beschreibt den Prozess des Grenzübertritts und der Grenzkontrolle mit Bezug auf Foucault als einen Übergangsritus, in 
dessen Zentrum ein „confessionary complex“ (Salter 2005, S. 44-46) stehe, im Zuge dessen Reisende sich dem Nationalstaat unterwerfen. Hier findet also eine Subjektivierung im wörtlichen Sinn statt. Grenzen und die Kontrolle derselben sind unerlässlich für die Herstellung und Imagination von Souveränität, und das relativ unabhängig von der Frage, inwieweit die Grenzkontrolle tatsächlich existiert, oder ob sie imaginiert wird: „This ever-increasing attempt to control human mobility tends to be promoted, in fact, as nothing less than a putative manifestation of these states' (,national') sovereign power" (De Genova 2010, S. 34).

Wenn die Grenze überall ist, und wenn die Grenzfunktion delokalisiert und deterritorialisiert ist, dann gilt dies ebenso für die Potenzialität des Ausnahmezustands und der Unsicherheit, die der nationalstaatlichen Grenze eingeschrieben ist. Dies gilt in besonderem Maße für Abschiebungen, wie De Genova zeigt. Er argumentiert, dass die scheinbar so klare Unterscheidung zwischen dem reinen Innen und dem bedrohlichen Äußeren empirisch nicht existiere und praktisch stets verschwimme:

"Indeed, there is hardly a more apt exemplar of this tendency than the anti-immigrant politics of nativism and hostility to ,foreign'-ness that commonly imbue regimes of deportability and deportation [...] Thus they effectively transform the entirety of the interior of any territorial space of ,national' community into an unrelenting regulatory sphere for migrants, a ,border' that is implosive, infinitely elastic, and in effect, truly everywhere within the space of the nation-state" (De Genova 2010, S. 52; Herv. i. O.).

Die Grenze ist zu jeder Zeit überall, aber das heißt nicht notwendigerweise, dass sie auch stets eindeutig ist. Dies zeigt sich im Diskurs über gesellschaftliche und ethnische Minderheiten. Minderheiten auf dem eigenen Staatsgebiet beispielsweise können von einer Mehrheitsgesellschaft als Sicherheitsbedrohung gewertet werden (vgl. Appadurai 2006; siehe auch Höfler/Klessmann in diesem Band). Potenziell stehen auch Minderheiten in Grenzregionen unter dem Verdacht der zweifelhaften Loyalität und der Präferenz für den benachbarten Nationalstaat. Zugleich können jedoch auch ethnische und kulturelle Minderheiten in den Verdacht geraten, den Nationalstaat aufgrund mangelnder Vertrauenswürdigkeit und zweifelhafter Loyalität zu destabilisieren, wie sich an den zwei folgenden Beispielen zum Entzug der Staatsbürgerschaft verdeutlichen lässt. Jüdinnen und Juden wurden in Nazi-Deutschland zwischen 1933 und 1945 nicht nur materiell enteignet, sondern zunächst auch zu Staatsbürger*innen zweiter Klasse, bevor die antijüdische Gesetzgebung der Nationalsozialisten sie ihrer Rechte, darunter der Staatsbürgerschaft, weitgehend beraubte (vgl. Weiss 2000). Dieser äußerst folgenreiche Akt ermöglichte es, Jüdinnen und Juden symbolisch sowie praktisch außerhalb der Grenzen der imaginierten Gemeinschaft zu positionieren, bevor sie als „abject or enemy citizens“ (De Genova 2010, S. 55) exkludiert und in die Vernichtungslager deportiert wurden.

Das zweite Beispiel zeigt die Aktualität der Staatsbürgerschaftsdebatte. In den USA hat die Trump-Regierung im Sommer 2018 angeordnet, jahrzehntealte Einbürgerungen im Hinblick darauf zu untersuchen, ob bei der Beantragung gelogen wurde (vgl. Gessen 2018). Fingerabdrücke, die seit den 1990er Jahren genommen wurden, sollen digitalisiert und mit denjenigen eingebürgerter Amerikaner*innen abgeglichen werden. Der Entzug der Staatsbürgerschaft droht Personen, die eine falsche Identität angegeben haben oder die nicht in allen Punkten wahrheitsgemäß geantwortet haben. Die Möglichkeit der Ausbürgerung wurde bislang vor allem auf schwere Verbrechen, wie z.B. nationalsozialistische Täter, angewandt. Mit der Trump-Regierung sollen die Tatbestände gravierend ausgeweitet werden. Die Washington Post führt das hypothetische, jedoch nicht unwahrscheinliche Beispiel einer Philippinin an, die ihr 
Arbeitsvisum überzieht, verhaftet und ausgewiesen wird. Jahre später heiratet sie einen Amerikaner und kehrt entsprechend mit neuem Namen in die USA zurück. Bei der Einbürgerung verschweigt sie die frühere Abschiebung: „Twenty years later, when the fingerprints from her first deportation are digitized, the woman is notified she will be stripped of her citizenship for lying on her application forms“ (Miroff 2018).

Eine Ausbürgerung stellt das Individuum außerhalb der Grenzen der Gemeinschaft. Dieser Prozess der Exklusion mit dem Argument eines Zugewinns an Sicherheit für die Mehrheitsgesellschaft bedeutet jedoch zugleich für das exkludierte Individuum einen Verlust von Sicherheit in einem weiten Sinn: Die Person verliert nicht allein ihre Arbeit, ihre Versicherungen, ihren Wohnort, ihr soziales Umfeld, sondern auch ihr Vertrauen in die Welt wird in ihren Grundfesten erschüttert. Ontologische Sicherheit, dass die Dinge sind, wie sie sind, und dass sie auch morgen noch so sein werden, die sogenannte Erwartungssicherheit, wird massiv gefährdet. Die Machtdemonstration des souveränen Nationalstaats, der seine Souveränität unter Beweis stellt, indem er außerhalb des Gesetzes handelt, steht dieser tiefen Verunsicherung diametral gegenüber.

\subsection{Akteur*innen der Grenzsicherheit}

Grenzschutz und Grenzkontrollen werden nicht allein (im Auftrag) von staatlichen Akteur*innen und Institutionen ausgeübt. Dem Fall der Kontrolle durch Dritte, wie Sicherheitsdienste oder Fluggesellschaften, nicht unähnlich, ist auch die Zivilgesellschaft aufgerufen, sich am Grenzschutz zu beteiligen. Dies wird insbesondere im Kampf gegen den internationalen Terrorismus sichtbar. Nick Vaughan-Williams (2008) zeigt, wie der Diskurs über Terrorismus Vorstellungen von Citizenship mit Anti-Terror-Initiativen verknüpft: Er berichtet von einer Plakatkampagne des London Metropolitan Police Service (Met) im Jahr 2004, kurz nach den Anschlägen in Madrid. Vaughan-Williams (ebd., S. 73f.) spricht vom „Citizen-Detective“, der mittels dieser Plakate zur proaktiven Mitarbeit aufgefordert ist. So zeigt eins der Poster ein anonymes Klingelschild und die Aufschrift: „Terrorists won't succeed if someone reports suspicious activity. You are that someone. “ Unter der Nummer einer „Anti-Terrorist Hotline“ findet sich noch die Erinnerung: „Terrorists need places to live. Are you suspicious of your tenants or neighbours?"“. Die weiteren Plakate der Kampagne sind in einem ähnlichen Stil gehalten und halten zur Wachsamkeit an; so sollen verdächtige Vorgänge rund um Garagen sowie an Flüssen oder auch bei Autokäufen o.Ä. beobachtet werden. Es geht also um Aktivitäten, die sich zwar allesamt im privaten Rahmen bewegen, laut Met jedoch ausdrücklich im öffentlichen Interesse sind. Damit sieht Vaughan-Williams (ebd., S. 68) die Bürger*innen in einer doppelten Rolle hinsichtlich der Praktiken der Überwachung: „as both objects of surveillance by authorities such as the police but also as agents of surveillance so that, effectively, citizens also become Europe's border guards.“

Im Londoner Beispiel suchen die Polizeibehörden die Bürger*innen proaktiv wie auch moralisch am Kampf gegen den Terror zu beteiligen und nehmen damit die Denunziation unbescholtener, jedoch aufgrund ihres Glaubens oder ihrer Herkunft per se verdächtiger Bürger*innen nicht allein billigend in Kauf, sondern fordern deren Securitization regelrecht ein. Indem die staatliche Kampagne auf die Terrorist*innen von nebenan hinweist, holt sie auch die Bedrohung vom Öffentlichen, Kollektiven ins Private und schafft damit eine persönliche 
Betroffenheit - und auch persönliche Verantwortung: „Thus, even as it warns of imminent security threats, the state seeks to reduce its own role in security provision through expanding individual ,reponsibilization“" (Goldstein 2010, S. 492).

Bürgerwehren sind weitere Beispiele, wo Bürger*innen selbst aktiv werden und domopolitics aktiv vorantreiben. Harel Shapira (2013) untersucht die sogenannten Minutemen, eine Art Bürger*innenwehr, die auf eigene Faust an der US-amerikanisch-mexikanischen Grenze patrouilliert und Grenzverletzungen meldet. Er zeigt in seiner sorgfältigen ethnografischen Studie, auf welch komplexe Weise Vorstellungen von Grenzziehungen, von Eigenem und vom Fremden hier existieren. Die Minutemen (und -women) sehen es als ihre patriotische Pflicht als Amerikaner*innen an, ihr Land vor ausländischen Drogenhändlern und Vergewaltigern zu schützen, die auch Donald Trump in seiner Präsidentschaftskampagne als Schreckgespenst heraufbeschworen hat. Zugleich stehen sie unter dem Druck von Bürger*innenrechtsorganisationen und liberals, die ihnen Rassismus vorwerfen, und die ebenfalls amerikanische Werte ins Feld führen. Zur Verhandlung steht die Frage, was dieses Amerikanische eigentlich ist, was alle Seiten zu verteidigen suchen. Die Gemeinschaft der Gleichgesinnten, das Zuhause, das höhere Gut wird von unterschiedlichen Akteur*innen beschworen, bedeutet aber jeweils etwas anderes. Entsprechend unterscheiden sich auch die Bedrohungen, vor denen geschützt wird. Die Grenzen verlaufen nicht nur am Rande des Territoriums, sondern quer durch die Gesellschaft.

Die Bevölkerung kann allerdings auch weit unvermittelter zum Sicherheitsakteur werden, und dies recht tatkräftig. Bereits mehrfach wurde von Flugpassagier*innen in den USA das Aussteigen vermeintlich muslimischer Mitreisender erzwungen. Pikanterweise waren in einem Fall die zwei betreffenden Imame auf dem Weg zu einer Konferenz zum Thema Vorurteile (Stern 2009; SPIEGEL Online 2011). Das Magazin Der Spiegel berichtete im Jahr 2011 von einer „arabisch aussehenden “ amerikanischen Hausfrau aus Ohio, die am 11. September 2011 gemeinsam mit ihren indisch aussehenden Sitznachbarn mit Handschellen und vorgehaltenen Maschinenpistolen auf dem Flughafen von Detroit aus dem Flugzeug eskortiert und stundenlang festgehalten wurde. FBI und Homeland Security teilten ihr mit, jemand „im Flugzeug habe gemeldet, dass sich die drei Passagiere in Reihe 12 verdächtig verhalten würden“, beispielsweise durch die Tatsache eines Toilettenbesuchs (Neufeld 2011).

Die diffuse Bedrohung durch den Terrorismus, gepaart mit einer diskursiven Verknüpfung mit Merkmalen der religiösen Zugehörigkeit oder einem ,arabischen' Erscheinungsbild schaffen eine Atmosphäre der gegenseitigen Verdächtigungen und stellen einen nicht unbeträchtlichen Teil der Gesellschaft unter Generalverdacht. Die Maßnahmen von Sicherheitsbehörden und Gesetzgeber*innen folgen nicht nur einer ähnlichen Logik, sondern formen den Interpretationsrahmen und den Diskurs, aus dem sich diese Vorstellungen speisen, mit. So haben die Anschläge in New York, Madrid, London, Nizza, Paris und Berlin zu umfangreichen rechtlichen und organisatorischen (Weiter-)Entwicklungen geführt, wie Austausch von Informationen und biometrischen Daten, Ausweitung und Neuschaffung von Datenbanken, Überwachungstechnologien, auf Ebene sowohl der EU als auch der Nationalstaaten. Die Entgrenzung des Kampfes gegen Gefahren wie den Terrorismus und die Ausweitung auf immer weitere gesellschaftliche Räume zeitigen die doppelte Rolle der Zivilgesellschaft im Komplex der Grenzsicherung. 


\section{Fazit}

Dieser Beitrag diskutierte den Zusammenhang von Grenzen und (Un-)Sicherheit und stellt heraus, dass in einer kulturanthropologischen Perspektive zwei grundlegende Prämissen von Bedeutung sind: zum einen ist Grenze nicht selbstevident und objektiv vorhanden, sondern sozial konstruiert und performativ; zum anderen zeigt sich der Zusammenhang von Grenze und (Un-)Sicherheit entsprechend in der gelebten Praxis. Dies beinhaltet den Alltag der Akteur*innen der Grenzsicherheit und die Heterogenität der alltäglichen Sicherheitspraktiken und Technologien, die nationalen und transnationalen Politiken, aber auch die Widerständigkeiten und Subvertierungen der Grenzsicherheit. Karine Côté-Boucher et al. (2014) weisen jedoch auch darauf hin, dass diese multitude möglicher Ausprägungen des Grenzsicherheitsdispositivs nicht dazu verleiten sollte, eine einheitliche Kraft oder Logik dahinter entdecken zu wollen. Sie verweisen deshalb auf die Notwendigkeit empirischer und praxeologischer Forschungen, um zu zeigen, auf welche Weise unterschiedliche Logiken und Rationalitäten koexistieren, die sich auf verschiedene Weise in die Praktiken und Diskurse der Akteur*innen der Sicherheit einschreiben, sich gegenseitig überlappen und auf diese Weise die Grenzen, Grenzsicherungspolitiken, die verschiedenen Akteur*innen und die beteiligten Institutionen einem permanenten Wandel unterziehen:

„Practice-based research constitutes an invitation to be cautious with an all-encompassing understanding of border security - expressing one main logic or rationality in all contexts, all practices, all security networks and institutions - a tendency within critical border and security scholarship that Walters (2011) aptly call the ,global security hypothesis “ (Côté-Boucher et al. 2014, S. 199).

Für kultur- und sozialwissenschaftliche Forschung bedeutet dies, dass Grenzen und Grenzziehungsprozesse in der Forschung nicht lediglich als gegeben angenommen werden sollen. Grenzen stiften Bedeutung, sie haben Handlungsmacht. Grenzen produzieren Unterschiede und schaffen soziale Räume, Hindernisse, Klassifikationen und Gelegenheiten. Die Grenzfunktion und die Grenzinfrastruktur sind machtvolle Faktoren. Kulturwissenschaftliche Forschung, die von der Lebenswelt der Akteur*innen ausgeht, muss die Kontingenzen einbeziehen, unter denen Handlung stattfindet, und die Narrative und Strategien, derer Akteur*innen und Institutionen sich bedienen. Deshalb sind Grenzen nicht nur Aktanten, sondern zugleich auch Objekte von Handlungsmacht. Sie werden angerufen, imaginiert und tragen symbolische Bedeutungen. Grenzen stellen einen wichtigen Aspekt sozialer Vorstellungen und Fantasien sowie von Prozessen der Ein- und Ausschließung dar. Die Imagination der Grenzfunktion und der Grenzinfrastruktur sind machtvolle Faktoren. In jedem Fall sind Grenzen soziale Fakten, die Differenz signalisieren und politische Effekte zeitigen.

Zukünftige Forschung sollte sich zunehmend darauf konzentrieren, auf welch unterschiedliche Weise Grenzen produziert und performiert werden, wie Akteur*innen Grenzziehungsprozesse mit Leben erfüllen, in ihre eigene Lebenswirklichkeit übersetzen oder subvertieren und welche Aussagen sich aus diesen Feldern wiederum über die übergreifende Frage nach dem Zusammenhang von staatlichem Handeln, transnationalen Prozessen und lokalen Vorstellungen zu safety und security machen lassen. Die greifbare Wirklichkeit und Wirkmacht von Grenzen zeigt sich insbesondere in einer Zeit, in der ein Diskurs vom grenzenlosen Europa von permanenten temporären Grenzkontrollen wieder eingeholt, wenn nicht überholt wird. Es scheint, dass auch fast hundert Jahre nach Max Webers berühmter Definition der Zusammenhang von 
Grenzen und vorgestellten Gemeinschaften auch im Zeitalter transkultureller Verflechtungen weiterhin hochaktuell bleibt.

\section{Weiterführende Literatur}

Donnan, Hastings/Wilson, Thomas M. (Hrsg.) (2010): Borderlands. Ethnographic Approaches to Security, Power, and Identity. Lanham/Boulder/New York/Toronto/Plymouth, UK: University Press of America.

Hall, Alexandra (2012): Border Watch: Cultures of Immigration, Detention and Control. Anthropology, Culture and Society. London: Pluto Press.

Löfgren, Orvar (1999): Crossing Borders: The Nationalization of Anxiety. In: Ethnologia Scandinavica 29, S. $5-27$.

Maguire, Mark/Frois, Catarina/Zurawski, Nils (Hrsg.) (2014): The Anthropology of Security: Perspectives from the Frontline of Policing, Counter-Terrorism and Border Control. London: Pluto Press.

\section{Literaturverzeichnis}

Agamben, Giorgio (1998): Homo Sacer. Sovereign Power and Bare Life. Stanford: Stanford University Press.

Ahmed, Sara (2000): Strange Encounters. Embodied Others in Post-Coloniality. London/New York: Routledge.

Anderson, Benedict (1998): Die Erfindung der Nation: zur Karriere eines folgenreichen Konzepts. Berlin: Ullstein.

Andersson, Ruben (2014): Illegality, Inc. Clandestine Migration and the Business of Bordering Europe. Oakland: University of California Press.

Andreas, Peter (2001): Border Games: Policing the U.S.-Mexico Divide. Ithaca: Cornell University Press.

Appadurai, Arjun (2006): Fear of Small Numbers. An Essay on the Geography of Anger. Durham/London: Duke University Press.

Appadurai, Arjun (2013): The Future as Cultural Fact. Essays on the Global Condition. London/New York: Verso.

Aradau, Claudia (2010): Security That Matters: Critical Infrastructure and Objects of Protection. In: Security Dialogue 41, H. 5, S. 491-514.

Baird, Theodore (2017): Knowledge of practice: A multi-sited event ethnography of border security fairs in Europe and North America. In: Security Dialogue 48, H. 3, S. 187-205.

Balibar, Étienne (2009): Europe as Borderland. In: Environment and Planning D: Society and Space 27, H. 2, S. 190-215.

Balzacq, Thierry (2011): A Theory of Securitization: Origins, Core Assumptions, and Variants. In: Balzacq, Thierry (Hrsg.): Securitization Theory. How Security Problems Emerge and Dissolve. London/New York: Routledge, S. 1-30.

Barth, Fredrik (Hrsg.) (1969): Ethnic Groups and Boundaries. The Social Organization of Culture Difference. Bergen/London: Allen \& Unwin.

Bauman, Zygmunt (2000): Liquid Modernity. Malden: Polity Press.

Beck, Ulrich (1986): Risikogesellschaft. Auf dem Weg in eine andere Moderne. Frankfurt/M.: Suhrkamp.

Bhabha, Homi K. (1994): The Location of Culture. London; New York: Routledge.

Bigo, Didier/Guild, Elspeth (2005): Policing at Distance: Schengen Visa Policies. In: Dies. (Hrsg.): Controlling Frontiers - Free Movement Into and Within Europe. Aldershot: Ashgate, S. 233-263.

Billig, Michael (1995): Banal Nationalism. London/Thousand Oakes/New Delhi: Sage.

Booth, Ken (1991): Security and emancipation. In: Review of International Studies 17, H. 4, S. 313-326.

Broeders, Dennis (2007): The New Digital Borders of Europe: EU Databases and the Surveillance of Irregular Migrants. In: International Sociology 22, H. 1, S. 71-92.

Brubaker, Rogers (2010): Migration, Membership, and the Modern Nation-State: Internal and External Dimensions of the Politics of Belonging. In: Journal of Interdisciplinary History XLI(1), S. 61-78.

Buckel, Sonja (2013): „Welcome to Europe“ - Die Grenzen des europäischen Migrationsrechts. Bielefeld: transcript Verlag.

Buzan, Barry/Wæver, Ole/Wilde, Jaap de (1998): Security: A New Framework for Analysis. Boulder: Lynne Rienner Pub.

Carey, Matthew/Pedersen, Morten Axel (2017): Introduction: Infrastructures of Certainty and Doubt. In: The Cambridge Journal of Anthropology 35, H. 7, S. 18-29.

Chavez, Leo R. (2007): The Condition of Illegality. In: International Migration 45, H. 3, S. 192-196.

Cohen, Anthony P. (1998): Boundaries and Boundary-consciousness: Politicizing Cultural Identity. In: Bort, Eberhard (Hrsg.): The Frontiers of Europe. London: Pinter, S. 22-35. 
Côté-Boucher, Karine/Infantino, Federica/Salter, Mark B. (2014): Border security as practice: An agenda for research. In: Security Dialogue 45, H. 3, S. 195-208.

Cunningham, Hilary/Heyman, Josiah (2004): Introduction: Mobilities and Enclosures at Borders. In: Identities: Global Studies in Culture and Power Special Issue: Movement on the Margins: Mobility and Enclosures at Borders 11, H. 3, S. 289-302.

Darling, Jonathan (2011): Domopolitics, governmentality and the regulation of asylum accommodation. In: Political Geography 30, H. 5, S. 263-271.

Darling, Jonathan (2014): Asylum and the Post-Political: Domopolitics, Depoliticisation and Acts of Citizenship. In: Antipode 46, H. 1, S. 72-91.

De Genova, Nicholas (2010): The Deportation Regime. Sovereignty, Space, and the Freedom of Movement. In: De Genova, Nicholas/Peutz, Nathalie (Hrsg.): The Deportation Regime: Sovereignty, Space, and the Freedom of Movement. Durham: Duke University Press, S. 33-65.

Douglas, Mary (1966/1984): Purity and Danger. An Analysis of the Concepts of Pollution and Taboo. London/New York: Routledge.

Eisch-Angus, Katharina (2011): „You Can't Argue with Security. “ The Communication and Practice of Everyday Safeguarding in the Society of Security. In: Behemoth - A Journal on Civilisation 4, H. 2, S. 83-106.

Feldman, Gregory (2012): The Migration Apparatus. Security, Labor, and Policymaking in the European Union. Stanford: Stanford University Press.

Follis, Karolina (2012): Building Fortress Europe: The Polish-Ukrainian Frontier. Philadelphia: University of Philadelphia Press.

Foucault, Michel (2006): Geschichte der Gouvernementalität 1: Sicherheit, Territorium, Bevölkerung: Vorlesung am Collège de France 1977/1978. Frankfurt/M.: Suhrkamp.

Friese, Heidrun (2017): Flüchtlinge: Opfer - Bedrohung - Helden. Zur politischen Imagination des Fremden. Bielefeld: transcript Verlag.

Furedi, Frank (2006): Culture of Fear Revisited. Risk-Taking and the Morality of Low Expectation. London/New York: Continuum.

Gessen, Masha (2018): In America, Naturalized Citizens No Longer Have an Assumption of Permanence. In: The New Yorker, 18.06.2018. www.newyorker.com/news/our-columnists/in-america-naturalized-citi zens-no-longer-have-an-assumption-of-permanence, 28.11.2019.

Giddens, Anthony (1990): The Consequences of Modernity. Cambridge: Polity Press.

Goldstein, Daniel M. (2010): Toward a Critical Anthropology of Security. In: Current Anthropology 51, H. 4, S. 487-517.

Götz, Irene (2011): Deutsche Identitäten. Wien: Böhlau.

Guiraudon, Virginie (2003): Before the EU border: Remote Control of the „Huddled Masses“. In: Groenendijk, Kees/Guild, Elspeth/Minderhout, Paul (Hrsg.): In Search of Europe's Borders. The Hague/London: Kluwer Law International, S. 191-214.

Guiraudon, Virginie (2006): Enlisting Third Parties in Border Control: a Comparative Study of its Causes and Consequences. In: Caparini, Marina/Marenin, Otwin (Hrsg.): Border and Security Governance. Managing Borders in a Globalised World. Wien: LIT, S. 79-96.

Gusterson, Hugh/Besteman, Catherine (Hrsg.) (2010): The Insecure American. How we got here and what we should do about it. Berkeley/Los Angeles/London: University of California Press.

Herlyn, Gerrit/Zurawski, Nils (Hrsg.) (2015): Achtung Sicherheitskontrollen! Flughäfen, Kultur und Un/ Sicherheiten. Münster: LIT Verlag.

Herzfeld, Michael (1997): Cultural Intimacy. Social Poetics in the Nation State. New York/London: Routledge.

Hess, Sabine/Kasparek, Bernd (Hrsg.) (2010): Grenzregime. Diskurse, Praktiken, Institutionen in Europa. Berlin: Assoziation A.

Houtum, Henk van/Naerssen, Ton van (2002): Border, Ordering and Othering. In: Tijdschrift voor Economische en Sociale Geografie 39, H. 2, S. 125-136.

Irek, Malgorzata (1998): Der Schmugglerzug: Warschau-Berlin-Warschau. Materialien einer Feldforschung. Berlin: Das Arabische Buch.

Johnson, Corey/Jones, Reece/Paasi, Anssi/Amoore, Louise/Mountz, Alison/Salter, Mark/Rumford, Chris (2011): Interventions on rethinking „the border“ in border studies. In: Political Geography 30, H. 2, S. 61-69.

Khosravi, Shahram (2010): „Illegal“ Traveller. An Auto-Ethnography of Borders. Basingstoke: Palgrave Macmillan.

Löfgren, Orvar (1989): The Nationalization of Culture. In: Ethnologia Europaea 19, S. 5-24.

Miroff, Nick (2018): Scanning immigrants' old fingerprints, U.S. threatens to strip thousands of citizenship. In: The Washington Post, 13.06.2018. www.washingtonpost.com/world/national-security/scan 
ning-immigrants-old-fingerprints-us-threatens-to-strip-thousands-of-citizenship/2018/06/13/2230d8a2-6 f2e-11e8-afd5-778aca903bbe_story.html?noredirect=on\&utm_term=.95addc41392a, 28.11.2019.

Neufeld, Dialika (2011): Gespenster. Warum eine amerikanische Hausfrau unter Terrorverdacht geriet. In: Der Spiegel 2011, H. 4, S. 56.

Rumford, Chris (2008): Introduction: Citizens and Borderwork in Europe. In: Space and Polity 12, H. 1, S. $1-12$.

Salter, Mark B. (2005): At the threshold of security: a theory of international borders. In: Zureik, Elia/Salter, Mark B. (Hrsg.): Global surveillance and policing: borders, security, identity. Cullompton: Willan, S. 36-50.

Salter, Mark B. (Hrsg.) (2008a): Politics at the Airport. Minneapolis/London: University of Minnesota Press.

Salter, Mark B. (2008b): When the exception becomes the rule: borders, sovereignty, and citizenship. In: Citizenship Studies 12, H. 4, S. 365-380.

Schwell, Alexandra (2008): Europa an der Oder: Die Konstruktion europäischer Sicherheit an der deutschpolnischen Grenze. Bielefeld: transcript Verlag.

Schwell, Alexandra/Eisch-Angus, Katharina (Hrsg.) (2018): Der Alltag der (Un)Sicherheit. Kulturwissenschaftlich-ethnographische Perspektiven auf die Sicherheitsgesellschaft. Berlin: Panama.

Shapira, Harel (2013): Waiting for José. The Minutemen's Pursuit of America. Princeton/Oxford: Princeton University Press.

SPIEGEL Online (2011): Muslime bitte aussteigen! In: SPIEGEL Online, 8.5.2011. www.spiegel.de/panora ma/gesellschaft/0,1518,761287,00.html, 10.10. 2018

Sheptycki, James (2002): In Search of Transnational Policing. Towards a sociology of global policing. Aldershot: Ashgate.

Stern (2009): Muslimische Passagiere müssen aussteigen. In: Stern, 2.1.2009. www.stern.de/politik/auslan $\mathrm{d}$ /pilot-hatte-sicherheitsbedenken-muslimische-passagiere-muessen-aussteigen-650329.html, 28.11.2019

Torpey, John C. (2000): The invention of the passport: surveillance, citizenship, and the state. Cambridge/New York: Cambridge University Press.

Vaughan-Williams, Nick (2008): Borderwork beyond Inside/Outside? Frontex, the Citizen-Detective and the War on Terror. In: Space \& Polity 12, H. 1, S. 63-79.

Walters, William (2004): Secure borders, safe haven, domopolitics. In: Citizenship Studies 8, H. 3, S. 237 260.

Walters, William (2006a): Border/Control. In: European Journal of Social Theory 9, H. 2, S. 187-203.

Walters, William (2006b): Rethinking Borders Beyond the State. In: Comparative European Politics 4, S. 141-159.

Weber, Eugen (1976): Peasants into Frenchmen. The Modernization of Rural France, 1870-1914. Stanford: Stanford University Press.

Weber, Max (1921/2005): Wirtschaft und Gesellschaft. Grundriss der verstehenden Soziologie. Frankfurt/M.: Zweitausendeins.

Weiss, Yfaat (2000): Deutsche und polnische Juden vor dem Holocaust. Jüdische Identität zwischen Staatsbürgerschaft und Ethnizität 1933-1940. München: Oldenbourg. 